\title{
Preface to the special issue of the "2nd Saudi-Chinese Oil Refinery Forum (SCORF)": advances in multiple catalytic strategies for producing cleaner fuels and petrochemicals
}

\author{
Zi-Feng Yan $^{1} \cdot$ Hamid A. Al-Megren ${ }^{2} \cdot$ Rui Feng ${ }^{1}$
}

Received: 29 July 2015/Accepted: 6 August 2015/Published online: 15 August 2015

(c) The Author(s) 2015. This article is published with open access at Springerlink.com

Energy resources, especially economical fossil fuels, are the foundation of fast economic development and ever-increasing wealth in the world over the past decades. For example, China has the world's second largest oil refinery and economic gross with a growing consumption of refined oil products, of 518 million tons of crude oil in 2014. Considering the broad consensus on this non-renewable resource and the environmental pollution caused by the use of fossil fuels, the pressure is on the government and oil refineries to seek and develop multiple catalytic strategies for producing cleaner fuels and the required petrochemicals.

The Saudi-Chinese Oil Refinery Forum (SCORF) provides a regular forum for scientists and engineers from academies and industries to get together and discuss the latest developments in oil refinery technologies, innovative catalysts and pollutant control in the refinery process. The inaugural SCORF was held in Saudi Arabia in 2013, organized by King Abdul Aziz City for Science and Technology (KACST). The subsequent annual forum was held in Qingdao, China, in 2014, organized by China University of Petroleum (CUP). The 2nd SCORF,

Zi-Feng Yan

zfyancat@upc.edu.cn

Hamid A. Al-Megren

almegren@kacst.edu.sa

Rui Feng

feng2007115@163.com

1 State Key Laboratory for Heavy Oil Processing, PetroChina Key Laboratory of Catalysis, University of Petroleum, 266580 Qingdao, China

2 Materials Science Research Institute, King Abdulaziz City for Science and Technology, P.O. Box 6086, Riyadh 11442, Kingdom of Saudi Arabia particularly the plenary and keynote speeches, emphasized the innovative materials for catalysts, environmentalfriendly refinery process, sulfur recovery technology and the integrations of refinery and process.

This special issue presents selected papers from the 2 nd SCORF 2014 pertinent to catalyst preparation and characterization, catalytic reactions, simulation computation, and new advances in petrochemical production and refining technologies. These papers have been subject to an initial screening process by the conference organizers and then a normal peer-review process. The following are some highlights as a general summary of the topics to cover in this issue.

The fluid catalytic cracking (FCC) process, as one of the largest petroleum processing units in oil refineries, converts heavy oil into beneficial intermediates such as propylene, gasoline and diesel. The crude oil supply has continuously become expensive in recent years; however, its quality has become heavier and inferior, showing lower cracking property and higher sulfur content. The paper titled "Desulfurization of Saudi Arabian crudes by oxidation-extraction method" presents the combination and optimization of oxidation and extraction to remove the sulfur from various oil fractions.

The primary target for oil refineries is to improve the conversion of the FCC feedstock. One of the most effective and efficient methods is the research and development of FCC catalyst active components. The papers titled "Preparation and characterization of hierarchical USY by post treatment" and "Preparation and catalytic performance of high dispersion of Y zeolite treated with alkali solution" present the importance of the textural and acid properties of Y zeolite of FCC catalyst in improving the conversion of heavy oil and product distribution. 
On the other hand, the strong industrial demands for high value propylene and butenes stimulates the research focus on it. The papers on "Synthesis of ZSM-5 zeolite from diatomite for fluid catalytic cracking (FCC) application" and "Synthesis of vanadium-based catalysts and their excellent catalytic behaviors on dehydrogenation of $\mathrm{C} 4$ hydrocarbons" introduce two methods to produce olefins.

To make up for the rising cost of petroleum and environment protection, oil refining industries are seeking to broaden their energy sources. Natural gas is a clean energy source, which has been put in ever-intensifying use due to its relative abundance. In addition to its use as a burning fuel, it is more efficient and economical to transform it to basic organic raw materials and further into high value petrochemicals via catalytic reactions. The papers titled "Ni catalysts with different promoters supported on zeolite for dry reforming of methane" and "Pore size-controlled synthesis of molecular sieves and their difference in the catalytic properties for Fischer-Tropsch synthesis" present the efficient utilization of methane via a series of catalytic reactions, converting $\mathrm{CH}_{4}$ into synthesis gas and valueadded transportation fuel.

To deal with the depletion of fossil energy in the future and the emission of carbon dioxide, new strategies have also been developed by broadening energy resources such as nuclear, wind power and bioenergy. The paper "Hydroliquefaction of woody biomass for bio-oil in supercritical solvent with [BMIM] $\mathrm{Cl} / \mathrm{NiCl}_{2}$ catalyst" presents a solution for substituting the usage of fossil fuel with biofuel from biomass, such as sawdust by biomass liquefaction in supercritical ethanol with ionic liquid as catalyst.

In the selected examples, most research focus on the catalysts/materials which manifests the importance of catalysts in the corresponding reactions. Various properties, such as metallic distribution, nanosize of active component, textural structure and acidity, must be considered first. On this basis, experimental conditions for catalytic reactions will be optimized in both bench scale and pilot platform before practical application. However, this process usually takes time and costs a lot for a series of tests. In recent decades, simulation computation method has been a more mature and applicable technique to describe the operating behavior in the FCC reactor due to high-performance computing technology. It is easier and economical for a refinery to adjust the process conditions referring to the simulating results. The papers titled "Ten lumped kinetic model for the two-stage riser catalytic cracking for maximizing propylene yield (TMP) process" and "Multi-scale CFD simulation of hydrodynamics and cracking reactions in fixed fluidized bed reactors" introduce a combination of simulation computation and the experimental data to predict the operating performance in practical application.

We hope that readers will find the range of research advances in multi-strategies covered in this special issue of Applied Petrochemical Research interesting, useful and inspiring to them. We appreciate all the authors and reviewers for their scientific contributions to the preparation and publication of this special issue. Last, but not the least, we would like to extend our sincere welcome to all readers to gather at the upcoming 3rd SCORF in 2015 in Saudi Arabia and discuss the advances in oil refinery and the inspiring scientific issues.

Open Access This article is distributed under the terms of the Creative Commons Attribution 4.0 International License (http:// creativecommons.org/licenses/by/4.0/), which permits unrestricted use, distribution, and reproduction in any medium, provided you give appropriate credit to the original author(s) and the source, provide a link to the Creative Commons license, and indicate if changes were made. 